\title{
Developmental changes in the engagement of episodic retrieval processes and their relationship with working memory during the period of middle childhood.
}

\author{
Sinead M. Rhodes, Derek Murphy, Peter J.B. Hancock \\ To appear in British Journal of Developmental Psychology
}

\begin{abstract}
We examined the development of children's engagement of the episodic retrieval processes of recollection and familiarity and their relationship with working memory (WM). Ninety-six children (24 in four groups aged 8, 9, 10, and 11 years) and twenty-four adults performed an episodic memory (EM) task involving Old/New, Remember/Know, and Source Memory judgments and numerous WM tasks that assessed verbal and spatial components of WM and delayed STM. Developmental changes were observed in EM with younger children $(8,9,10$ year olds) making fewer Remember responses than 11-year-olds and adults while 11-year-olds did not differ from adults. Only children aged 10 years plus showed a relationship between EM and WM. EM was related to verbal executive WM in 10 and 11-year-old children suggesting that children at this stage use verbal strategies to aid EM. In contrast EM was related to spatial executive WM in adults. The engagement of episodic retrieval processes appears to be selectively related to executive components of verbal and spatial WM, the pattern of which differs in children and adults.
\end{abstract}

Keywords: episodic memory, memory retrieval, working memory, episodic buffer, executive functions, verbal, spatial, child development.

\section{Introduction}

Research investigating developmental changes in the engagement of the episodic retrieval processes of recollection and familiarity is relatively limited. Studies conducted to date have reported distinct developmental trajectories for the development of recollection and familiarity in children. Developmental changes have been reported in the engagement of recollection across middle childhood and into adolescence (Billingsley, Smith \& McAndrews, 2002; Brainerd, Holliday \& Reyna, 2004; Defeyter, Russo, McPartlin, 2009; Ghetti \& Angelini, 2008; Holliday, 2003). The specific age at which children engage recollection to the same degree as adults is however as yet unknown. The nature of the relationship between episodic retrieval processes and working memory is also limited. Recent research within the adult literature has reported interactions between the two; early stage maintenance in working memory has been reported to predict subsequent long-term memory retrieval (Ranganath, Cohen \& Brozinsky, 2005). The current study set out to investigate developmental changes in the engagement of episodic retrieval processes at specific ages within the period of middle childhood and to examine the relationship between episodic memory and a range of aspects of working memory.

\subsection{Developmental changes in episodic retrieval processes}

While children's accuracy in their memories for previous events has been the focus of considerable investigation within the psychological literature, most research has tended to focus on the quantitative rather than the qualitative aspects of memory performance which are in fact 
thought to be more important in every day life situations (Roderer \& Roebers, 2009). Knowledge of developmental changes in the engagement of the specific episodic retrieval processes of familiarity and recollection is relatively limited which is surprising given the wealth of research that has investigated the engagement of episodic retrieval processes in adults (Rhodes \& Donaldson, 2007, 2008; Yonelinas, 2002). Popular dual process theories of episodic memory (e.g. Atkinson \& Joula, 1973; Jacoby 1991; Mandler, 1980; Tulving, 1985) explain familiarity and recollection as two distinct processes. Familiarity is a relatively automatic process involving recognition without the retrieval of contextual information, such as the common experience of meeting someone on the street and feeling that you know them without being able to recollect any specific details about them. Recollection, in contrast, is a more controlled process that supports retrieval of information and its context (Yonelinas, 2002). Research conducted with children has suggested developmental changes in the use of recollection and familiarity to support retrieval during childhood.

A number of studies have reported distinct developmental trajectories for recollection and familiarity (e.g. Brainerd et al., 2004; Ghetti \& Angelini, 2008). Employing the conjointrecognition procedure (CRP), Brainerd et al. (2004) reported that use of recollection to support retrieval was 2 to 3 times higher in 14-year olds than 7-year olds whereas familiarity was stable across this period. Ghetti and Angelini (2008) used the Receiver Operating Characteristics Curves method (ROC) to calculate the engagement of recollection and familiarity and reported a similar pattern of engagement of episodic retrieval processes from 6 to 18 years old. Children aged 8, 10, 14 and 18 did not differ in engagement of familiarity while developmental changes were observed in recollection. While 6 and 8 year olds did not differ, use of recollection was lower in both age groups in contrast to 10 and 18 year olds and 6 year olds additionally differed from 14 year olds.

Another approach to measure episodic retrieval processes has been the comparison of item and source memory judgments. Cycowicz, Friedman, Snodgrass, \& Duff (2001) compared item and source recognition memory performance for children aged 7-8 years and college students. Performance differences between children and adults on an item recognition task that encouraged the use of familiarity was observed but was less pronounced than recollection differences (Cycowicz et al., 2001). In a further study the authors compared item and source memory in children aged 9-10 and 12-13 while event-related potentials (ERPs) were recorded (Cycowicz, Friedman \& Duff, 2003). While the ERP effect commonly associated with recollection (known as a parietal old/new effect) was elicited in both item and source memory tasks for both groups of children, suggesting that recollection was used as a basis of memory judgments for all age groups, the topography of the effect differed according to age group during performance of the source memory task alone. This finding suggests that children and adults rely on different neuronal networks in the retrieval of source information.

As has been noted in recently published work (Ghetti \& Angeleini, 2008; Roderer \& Roebers, 2009) the qualitative or subjective aspect of children's memory has been relatively ignored in the literature. Billingsley et al. (2002) incorporated qualitative measures of episodic retrieval and the findings reported for developmental changes in recollection generally converge with those assessed using quantitative methods. Billingsley et al., (2002) employed a Remember/Know task (Tulving, 1985; see Yonelinas, 2002) which provides an index of familiarity ('Know' responses) and recollection with familiarity ('Remember' responses) and reported that 8-10 year old children had a lower proportion of Remember responses than adolescent participant groups aged 14-16 and 17-19 years. Findings relating to the engagement of familiarity within the Billingsley study show slight differences from other studies. In contrast to Ghetti and Angelini (2008) and Brainerd et al. (2004) who reported no further changes in 
familiarity beyond the age of 8, Billingsley and colleagues (2002) reported that 8-10 year olds had a higher proportion of correct Know responses than the adolescent groups. Know responses were at floor for all groups, however, making it difficult to make definitive conclusions regarding familiarity differences between age groups. The study may have also been underpowered to detect significant effects across all age groups as each group comprised just 13 children. For example, it is difficult to ascertain whether a lack of significant differences reported in the proportion of Remember and Know responses between 8-10 year olds and 11-13 year olds reflects power issues or a lack of developmental changes during these time points. The pattern of data reported graphically (see Billingsley, 2002) indeed suggests that a lack of power may underlie the nonsignificant changes between these age groups.

Thus, a growing body of research suggests distinct developmental trajectories for recollection and familiarity during childhood and there is consensus that developmental changes in the use of recollection continues across the period of middle childhood and into adolescence. Importantly, these consistent findings have been reported across tasks with very different parameters with both quantitative and qualitative indices of episodic retrieval processes $(\mathrm{R} / \mathrm{K}$, ROC, CRP, ERP). Collectively, these studies suggest that children continue to show developmental changes in the use of recollection across the period of middle childhood and develop adult levels of engagement at some stage between the age of 8 years and early adolescence. The current study aimed to identify the specific age at which children show adult levels of recollection and to document possible corresponding developmental changes in the engagement of familiarity during middle childhood. It is predicted that the proportion of responses associated with recollection will increase across this period of middle childhood accompanied by a relative decrease in familiarity based responding. As research utilizing subjective measures of episodic retrieval is extremely limited an $\mathrm{R} / \mathrm{K}$ task was used to assess the engagement of recollection and familiarity in children. This $\mathrm{R} / \mathrm{K}$ task additionally incorporated a source memory judgment following Remember responses to provide an objective record of children's memory for source information.

\subsection{The relationship between episodic retrieval and working memory in children}

It is well established that working memory abilities undergo profound changes during the period of childhood up until the period of adolescence (Luciana \& Nelson, 1998; Welsh, Pennington \& Grossier, 1991). While various models of working memory have been proposed (e.g. Baddeley, 1986; Miyake, Friedman, Rettinger, Shah, \& Hegarty, 2001; Shah \& Miyake, 1996) the weight of evidence supports Baddeley's theoretical working memory component model (Baddeley \& Hitch, 1974; Baddeley, 1986, 2006). This model identifies verbal (phonological loop) and spatial (visuo-spatial sketchpad) working memory components which are involved in the maintenance of information in short-term memory and a 'central executive' where stored information is controlled and manipulated. The relationship between the engagement of episodic retrieval processes and working memory functioning is of particular interest because the ability to encode and retrieve from long-term memory ultimately depends on working memory abilities used to encode information into long-term memory.

The link between working memory and long term memory is a current hot topic in memory research, arising from the revision of Baddeley's working memory model to incorporate an episodic buffer, a system that is described as forming "an interface between the three working memory subsystems and long-term memory" (Baddeley, 2007, p13). Recent research within the adult literature has reported interactions between episodic memory and working memory. Ranganath and colleagues (2005) reported that early stage maintenance in working memory 
predicts subsequent long-term memory retrieval. Research investigating the relationship between episodic memory and working memory in children is limited. The current study aimed to investigate the relationship between a range of aspects of working memory including verbal and spatial working memory components assessing both storage and central executive aspects. As a number of studies have reported a link between executive functions and long-term memory in children (Drummey \& Newcombe, 2002; Sluzenski, Newcombe \& Ottinger, 2004) it is predicted that central executive aspects of working memory will in particular relate to episodic retrieval in children.

\subsection{The present study}

The present study had two principal aims. The first aim was to investigate developmental changes in children's engagement of familiarity and recollection at specific ages during the period of middle childhood. A second aim was to examine the relationship between the engagement of episodic retrieval processes and working memory which is known to develop during childhood. The study aimed to relate episodic memory retrieval to verbal and spatial storage and central executive aspects of working memory. The study further investigated the relationship between episodic memory and delayed short-term memory to examine the relationship between the ability to hold information in memory over time in short-term memory and episodic retrieval from longterm memory. The developmental time points that were assessed covered a range of specific ages within the period of middle childhood (8-11 years). This period was chosen based on a lack of research examining changes in episodic retrieval processes at specific time points between the ages of 8 and pre-adolescence.

\section{Participants}

\section{Method}

One hundred and thirty-five participants were recruited to the study: 111 children and 24 adults. All children recruited to the study were screened with the Teacher rated Strengths and Difficulties Questionnaire (SDQ) [Goodman, 2001] to ensure that the child was not suffering from any mental or behavioural disorder that could affect memory performance e.g. the common developmental disorder ADHD (Rhodes, Coghill \& Matthews, 2005). A total of 96 children were rated as symptom free $(\mathrm{T}$-score $<60)$ and were included in the study $(\mathrm{N}=24$ each group). The four child groups were matched on British Picture Vocabulary Scale (BPVS) Percentile Rank, an index of verbal ability $(\mathrm{F}<1, \mathrm{p}>.05)$ and gender (see Table 1). Parents of all participating children provided consent for their child to take part in the study in line with Departmental ethics regulations that was secured for the study.

Table 1

Participant Characteristics

\begin{tabular}{lll}
\hline $\begin{array}{l}\text { Age } \\
\text { (Children: years; }\end{array}$ & BPVS Percentile Rank & Gender \\
Adults: mean, s.d., range) & M (SD) & \\
\hline 8 & $64.6(19.0)$ & $12 \mathrm{M}, 12 \mathrm{~F}$ \\
9 & $64.3(18.2)$ & $12 \mathrm{M}, 12 \mathrm{~F}$ \\
10 & $60.0(21.9)$ & $13 \mathrm{M}, 11 \mathrm{~F}$ \\
11 & $66.6(22.8)$ & $12 \mathrm{M}, 12 \mathrm{~F}$ \\
Adults: 24, 5.0, 17-37 & N/A & $12 \mathrm{M}, 12 \mathrm{~F}$ \\
\hline
\end{tabular}




\section{Materials and Procedure}

The British Picture Vocabulary Scale (BPVS $2^{\text {nd }}$ Edition) [(Dunn, Dunn, Whetton \& Burley, 1997], an individually administered, norm-referenced, wide-range test of receptive vocabulary for Standard English was used to estimate general intellectual ability. The child groups were matched on BPVS Percentile Rank scores to ensure equivalent general intellectual ability (relative to age) across groups.

\section{Episodic Memory Task}

All participants performed a Remember/Know $(\mathrm{R} / \mathrm{K})$ task to assess the engagement of episodic retrieval processes. The task was created using the experimental program Eprime. In study phases of the experiment, participants were shown pictures presented on either the left or right hand side of the screen. The pictures comprised concrete objects based on common objects normed for children and adults (Snodgrass \& Vanderwart, 1980). Each study image was preceded by a fixation cross which appeared on the screen for 2 seconds duration. Each study image was presented for 3 seconds duration and participants were instructed to try and remember each image and the location on the screen it was presented. In test phases, participants were presented either with studied (old items) or unstudied (new items) pictures presented at central fixation for 3 seconds duration. Participants were required to make a three part response firstly indicating whether the item was old or new. If the item was judged to be old the participant was required to indicate whether they remember, knew, or merely guessed that the item was old. If the item was judged to be new participants were presented with the next test image. The guess option was included because it was anticipated that young children would mistake 'Know' judgments for guessing. The inclusion of a Guess response is thought to make Know judgments less liberal and more accurate (Knott \& Dewhurst, 2007). Finally, if the participants had chosen the Remember option they were presented with a third set of responses and were required to indicate whether the stimulus was presented at the left or right hand side of the screen. A third option 'something else' was included in case the participant didn't remember the specific location but had a specific recollection about the image (e.g. one child commented that "I remember seeing the snowman, because it was the first picture I saw in the Study Phase"). This task thus provided an index of familiarity ('Know' responses) and recollection with familiarity ('Remember' responses) in addition to a measure of memory for source. Each question remained on the screen until the participant responded. Participants completed a practice block comprising 4 pictures at study and 8 (4 old, 4 new) at test. The main part of the experiment comprised four study-test blocks. There were 16 pictures presented at each study phase and 32 (16 old, 16 new) presented at test phases.

\section{Working Memory Tasks}

Participants performed a range of working memory tasks: verbal and spatial storage and executive WM tasks and a delayed short-term memory task. Three of the tasks were taken from the Cambridge Neuropsychological Test Automated Battery (CANTAB) (Morris, Evendon, Sahakian, \& Robbins, 1987): the Spatial Span, Spatial Working Memory, and Delayed Matching to Sample tasks. These tasks have been extensively validated in both child and adult populations (Curtis, Lindeke, Georgieff \& Nelson, 2002; Luciana \& Nelson, 1998; Rhodes, Coghill, Matthews, 2004, 2005, 2006; Robbins, James, Owen, Sahakian, McInnes, \& Rabbitt, 1994) and typical developmental trajectories of performance have been reported (Curtis et al., 2002; Luciana \& Nelson, 1998; Robbins et al., 1994). The components of verbal working memory were assessed 
using working memory tasks previously reported in the literature that have been designed to tap a) verbal short-term memory (maintenance of information i.e. tapping the phonological loop), and b) verbal executive working memory (maintenance and manipulation of information i.e. tapping the phonological loop + central executive). These tasks have been adapted by the lead author for use with children based on tasks used in a number of published studies (Cannon, Glahn, Kim, Van Erp, Karlsgodt, Cohen, Nuechterlein, Bava, \& Shirinyan, 2005; D’Esposito, Postle, Ballard, \& Lease, 1999; Kim, Glahn, Nuechterlein \& Cannon, 2004). Specific task parameters are described below.

\section{Short-Term Memory storage tasks}

The storage components of working memory were assessed using the verbal short-term memory (STM) component task and the Spatial Span task from the CANTAB battery. The verbal STM component task assesses the ability to maintain a string of 3 letters in memory (target) which is displayed on the screen for 2.5 seconds followed by a 6 second delay (in which a fixation cross is displayed). The participant is then required to decide whether the probe presented is a 'match' or 'non-match' to the target (Canon et al., 2005; D'Esposito et al., 1999; Kim et al., 2004). The Spatial Span task is a test of spatial short-term memory capacity based on the Corsi block-tapping task (Milner, 1991) which assesses a participant's ability to remember the spatial locations of a sequence of squares on a computer screen without placing demands on central executive functioning. The key measure on this task is the Spatial Span Score. A participants' Spatial Span is defined as the longest sequence that they could reproduce correctly within three attempts. Executive working memory (WM) tasks

The central executive components of WM were assessed using the verbal executive WM component task and the Spatial Working Memory task from the CANTAB battery. The executive verbal WM task is similar in parameters to the verbal STM component task but in addition to assessment of maintenance of information this task also requires the participant to manipulate the information held in the working memory (Baddeley, 1986). In the verbal executive task, participants had to put the letters displayed in alphabetical order in their minds and hold this manipulated version in their maintenance store during the delay. The target letters were displayed for 2.5 seconds and the delay that followed comprised a 6 second interval during which a fixation cross was displayed. The participant then had to decide whether the probe presented was a 'match' or 'non-match' of the manipulated version (Canon et al., 2005; D'Esposito et al., 1999; Kim et al., 2004). The Spatial Working Memory (SWM) task places similar places demands on central executive functioning. It is a self-ordered searching task (Petrides \& Milner, 1982) task that assesses the participant's ability to retain spatial information and to simultaneously store and manipulate information in working memory while working towards a goal. Participants are required to 'search through' a spatial array of coloured boxes presented on the screen to collect 'blue tokens' hidden inside the boxes. Returning to a box where a token has already been found constitutes a 'Between Search Error' (BSE). Participants must keep searching through all the boxes until they find the blue token at which point they proceed to find the next hidden blue token. Ultimately participants will find a blue token behind each of the boxes. Experimental trials commence with a 4 box search and the highest difficultly level involves 8 box trials. Participants can use a (self-initiated) strategy to aid performance, for example always starting at top left of the array of boxes moving across to bottom right. A Strategy Score is calculated based on how often a searching sequence was initiated from the same box during a trial (Fray \& Robbins, 1996). A higher Strategy Score therefore indicates a lower use of strategy. 


\section{Delayed STM}

The Delayed Matching to Sample task assesses the ability to remember the visual features of a complex abstract target stimulus and to select from a choice of four patterns after a variable delay (patterns appear immediately in a ' 0 delay' condition, or after 4 , or 12 seconds). This task provides an index of delayed short-term functioning.

\section{Procedure}

The participants performed the tasks across 3 sessions with the order of tasks counterbalanced. These sessions were timetabled approximately 1 week apart.

The CANTAB tasks were presented on a high-resolution colour monitor utilizing a touch sensitive screen. The working memory and $\mathrm{R} / \mathrm{K}$ tasks were performed on the same machine which participants performed using a stimulus-response box.

\section{$R / K$ task training and instructions}

All participants received training on the $\mathrm{R} / \mathrm{K}$ task at the beginning of the testing session that involved the episodic memory task. Children received extensive training as to what the $\mathrm{R} / \mathrm{K}$ procedure involved and the experimenter ensured all children understood the task instructions before commencing testing whereby each child provided clear examples to verify their understanding. The $\mathrm{R} / \mathrm{K}$ task was performed using an SR box with buttons labelled to indicate the responses (Old/New; Remember/Know/Guess, Left/Right/Something Else). Participants first performed a practice testing session with the task requirements described prior to the study and test phases as appropriate. Instructions for $\mathrm{R} / \mathrm{K}$ judgments were adopted from Gardiner and Richardson-Klavehn (2000). Prior to the practice test phase the experimenter asked the child if they could explain the difference between "Knowing" and "Remembering" a picture. At this stage, most of the children could explain the difference between the two. Any children who were not perfectly clear as to the difference at this stage were given a further explanation whereby the experimenter used examples from everyday life. In this case the experimenter would ask "have you ever seen somebody and you knew them from somewhere, but you couldn't remember their name, or where you had seen them before? You might think "I know him from somewhere, but I can't remember where", but other times you see someone and might remember "oh, that's John from school, he sits on my left in class..., so you'd say that you remember him." After the practice test phase, the experimenter again asked each participant to explain the difference between 'Remember' and 'Know' relating to the pictures they had just responded to.

\section{Statistical Analysis}

All analyses were conducted using SPSS for Windows (v.16, SPSS Inc. Chicago, Ill.).

Episodic memory data were analysed using ANOVAs with a between subject factor of group (with 5 levels: 8, 9, 10, and 11-year-olds, and adults) conducted separately on hits, correct rejections, false alarms, Remember responses, Know responses, Guess responses and Left/Right judgments. Analysis of Remember and Know responses were conducted in line with similar studies with children (e.g. Billingsley et al., 2002). Thus, Remember, Know, and Guess responses were first adjusted to remove those arising from false positives and then calculated as a proportion of hits rather than of the total (correct and incorrect) of old/new responses. Left/Right responses are reported as a proportion of corrected Remember responses. Signal detection measures d-prime (sensitivity) and criterion were also calculated and were subjected to ANOVAs comparing group 
performance. Working memory data were also analyzed with factor of group. On all measures only significant effects ( $\mathrm{p}<.05$ ) for main effects of group and interactions related to within subject variables included in the repeated measures design (e.g. difficulty level SWM) are reported. Analyses on the Delayed Matching to Sample task were conducted separately on simultaneous and delay conditions in line with other studies (e.g. Rhodes et al., 2004, 2005, 2006). Data from repeated measures ANOVAs report degrees of freedom and F values with the Greenhouse Geisser correction where appropriate. Following ANOVA, LSD post-hoc analysis was used, with $\alpha=0.05$ unless otherwise indicated. Bivariate Pearson correlations were conducted between significant episodic memory and WM measures with $\alpha=0.01$ to account for multiple comparisons. Mean test scores and main statistics on the episodic memory and working memory tasks are presented in Table 2 and significant correlations are shown in Table 3.

\section{Episodic Memory Data}

\section{Results}

\section{Accuracy}

An ANOVA on hits for old responses revealed a significant effect of group $[\mathrm{F}(4,119)=3.99$, $\mathrm{p}<.005]$. Post-hoc tests revealed that 8 year olds had significantly lower hits than 11-year-olds $(\mathrm{p}<.01)$ and adults $(\mathrm{p}<.001)$. Adults also had significantly greater hits than 9 -year-olds $(\mathrm{p}=.05)$ and 10 -year-olds $(\mathrm{p}<.01)$. Analyses of correct rejections and false alarms revealed no significant effect of age group. Analyses of signal detection measures revealed significant group differences for dprime sensitivity $[\mathrm{F}(4,119)=5.92, \mathrm{p}<.001)$ but not criterion. Sensitivity was poorer in 8 -yearolds than 9 and 11-year-olds and adults and indeed all child groups with the exception of 11-yearolds were poorer in sensitivity than adults (see Table 2).

An ANOVA on corrected Remember responses revealed a significant effect of group $[\mathrm{F}(4,119)=34.01, \mathrm{p}<.004]$. Post-hoc analysis revealed that adults made significantly more Remember responses than 8,9, and 10-year-olds (all $\mathrm{p}<.01$ ) but there was no significant difference between 11-year-olds and adults. Eleven-year-olds made more Remember responses than 10-yearolds ( $\mathrm{p}<.05)$. An ANOVA on corrected Know responses revealed a significant effect of group $[\mathrm{F}(4,119)=2.91, \mathrm{p}<.03]$. Post-hoc analysis revealed that adults made significantly fewer Know responses than 10-year-olds ( $\mathrm{p}<.001)$, with a trend for fewer responses for adults than 9-year-olds $(\mathrm{p}=.07)$ and 8 -year-olds $(\mathrm{p}=.087)$ but there was no significant difference between 11 -year-olds and adults. Eleven-year-olds gave fewer Know responses than 10-year-olds. An ANOVA on corrected Guess responses revealed a significant effect of group $[\mathrm{F}(4,119)=4.33, \mathrm{p}<.003]$. Post-hoc analysis revealed that 8-year-olds made more Guess responses than 10 and 11-year-olds and adults (all $\mathrm{p}<.05)$. Adults made fewer Guess responses than 8 and 9year-olds $(\mathrm{p}<.05)$ and did not differ from 10 and 11-year-olds. There was, however, no significant effect of group on left/right accuracy judgments, calculated as a proportion of genuine Remember responses $[\mathrm{F}(4,119)=2.01$, $\mathrm{p}>.05]$. Summary data are reported in Table 2. In summary, developmental changes in the engagement of episodic retrieval processes were observed across the period of middle childhood with 11-yearolds performing similarly to adults on some episodic measures.

\section{WM Data}

\section{WM Component Tasks: Short-Term Storage}

An ANOVA on the verbal STM component task revealed a significant effect of group [F $(4$, $119)=13.03, \mathrm{p}<.001]$. Post-hoc tests revealed developmental improvements across all age groups (all $\mathrm{p}<.05$ ) with the exception of non-significant differences between 8 and 9-year-olds and 10 and 11-year-olds (all p>.05). 
An ANOVA on span length on the Spatial Span (SSP) task revealed a significant effect of group $[\mathrm{F}(4,119)=7.68, \mathrm{p}<.001]$. Post-hoc tests revealed that 8 -year-olds had a shorter span length than 11-year-olds and adults. The adult group had a significantly greater span length than all of the child age groups (all $\mathrm{p}<.01$ ). In summary, children showed developmental changes in storage aspects of verbal and spatial WM, with all child age groups showing poorer performance to adults. WM Component Tasks: Executive

An ANOVA on accuracy data on the verbal executive WM component task revealed a significant effect of group $[\mathrm{F}(4,119)=18.0, \mathrm{p}<.001]$. Post-hoc tests revealed developmental improvements across all age groups (all $\mathrm{p}<.05)$ with the exception of non-significant differences between 8 and 9-year-olds, 9 and 10-year-olds, and 10 and 11-year-olds (all p>.05).

A repeated measures ANOVA on between search errors on the Spatial Working Memory (SWM) task including a within subject factor of difficulty level $(4,6,8$ boxes) revealed a significant effect of group $[\mathrm{F}(4,110)=20.84, \mathrm{p}<.001]$ and a significant interaction between group and difficulty level $[\mathrm{F}(5.9,162.5)=11.76, \mathrm{p}<.001]$. Post-hoc tests revealed that the effect of group reflected developmental improvements in working memory across the age groups (all $\mathrm{p}<.05$ ) with the exception of non-significant differences between 9, 10 and 11-year-olds (all $\mathrm{p}>.05)$. The significant interaction between difficulty level and group was followed up with separate ANOVAs at each level with associated follow-up post-hoc tests. An ANOVA at the 4 box stage revealed a significant effect of group $[\mathrm{F}(4,119)=3.62, \mathrm{p}<.008]$. Post-hoc tests revealed that 8 -year-olds made more between search errors than 10 and 11 -year-olds and adults (all $\mathrm{p}<.05$ ). An ANOVA at the 4 box stage revealed a significant effect of group $[\mathrm{F}(4,119)=3.62, \mathrm{p}<.008]$. Posthoc tests revealed that 8-year-olds made more between search errors at the 4 box stage than 10 and 11-year-olds and adults and adults additionally showed fewer errors to 9-year-olds (all $\mathrm{p}<.05)$. An ANOVA at the 6 box stage revealed a significant effect of group $[\mathrm{F}(4,119)=15.06, \mathrm{p}<.001]$. Posthoc tests revealed that 8-year-olds made more between search errors at the 6 box stage than 10 and 11-year-olds and adults. The adult group made fewer errors than all of the child age groups (all $\mathrm{p}<.05)$. An ANOVA at the 8 box stage revealed a significant effect of group $[\mathrm{F}(4,119)=17.6$, $\mathrm{p}<.001]$. Post-hoc tests revealed that 8-year-olds made more between search errors at the 8 box stage than all other child groups and adults (all $\mathrm{p}<.05$ ). The adult group made fewer errors than all of the child age groups (all $\mathrm{p}<.05$ ), see Table 2.

An ANOVA on Strategy Score on the SWM task revealed a significant effect of group $[\mathrm{F}(4,119)=14.8, \mathrm{p}<.001]$. Post hoc tests revealed that 8 -year-olds had a higher Strategy Score indicating a lower use of strategy than 10-year-olds and adults (all $\mathrm{p}<.05$ ) and 9-year-olds had a higher strategy score than 10-year-olds and adults (all $\mathrm{p}<.05$ ). The adult group had a lower Strategy Score than all of the child age groups (all $\mathrm{p}<.05$ ). In summary, developmental changes in verbal and spatial executive WM and strategy use to support WM were observed across the age groups with all child age groups showing poorer performance to adults. Younger children (8 and 9 -year-olds) also seemed to rely less on using a strategy than older children and adults.

\section{Delayed short-term memory}

An ANOVA on percentage of correct responses during the simultaneous condition of the Delayed Matching to Sample (DMtS) task revealed no significant main effect of group ( $\mathrm{p}>.05)$. A repeated measures ANOVA on percentage of correct responses during the delay conditions $(0,4,12 \mathrm{~ms})$ of the Delayed Matching to Sample task revealed a significant main effect of group $[\mathrm{F}(4,110)=10.6$, $\mathrm{p}<.001]$. Post-hoc tests revealed developmental changes with increased accuracy with increasing age across age groups (all $\mathrm{p}<.05$ ) with the exception of no significant differences between 8 and 9 year-olds, 9 with 10 and 11-year-olds, and 11-year-olds compared to adults (all p>.05), see Table 
2. This data suggests that children have the ability to hold information in memory over a delay at adult levels by age 11 .

\section{Table 2}

Summary of episodic and working memory data

\begin{tabular}{|c|c|c|c|c|c|c|c|c|}
\hline Measure & 8 & 9 & 10 & 11 & Adult & $\mathrm{F}$ value & $\mathrm{p}$ value & Post-hoc summary \\
\hline Hits & 78 & 84 & 82 & 87 & 91 & 3.99 & $\mathrm{p}<.005$ & $\begin{array}{l}8<11 \text { and adults } \\
\text { Adults }>8,9,10\end{array}$ \\
\hline Correct Rejections & 91 & 94 & 93 & 94 & 96 & 1.62 & NS & NS \\
\hline False Alarms & 5.6 & 3.5 & 4.5 & 3.9 & 2.5 & 1.62 & NS & NS \\
\hline $\begin{array}{l}\text { Remember } \\
\text { responses* }\end{array}$ & 63 & 65 & 61 & 74 & 83 & 4.01 & $\mathrm{p}<.007$ & $\begin{array}{l}11>10 \\
\text { Adults }>8,9,10\end{array}$ \\
\hline Know responses* & 24 & 25 & 33 & 20 & 15 & 2.91 & $\mathrm{p}<.03$ & Adults, $11<10$ \\
\hline Guess responses* & 12.8 & 9.6 & 5.5 & 5.5 & 1.7 & 4.33 & $\mathrm{p}<.003$ & $\begin{array}{l}8>10,11, \text { adults } \\
\text { Adults }<8,9\end{array}$ \\
\hline L/R accuracy* & 82 & 91 & 84 & 85 & 93 & 2.01 & NS & NS \\
\hline d-prime & 2.3 & 3.0 & 2.7 & 3.1 & 3.5 & 5.92 & $\mathrm{p}<.001$ & $\begin{array}{l}8<9,11, \text { and adults } \\
\text { Adults }>8,9,10\end{array}$ \\
\hline Criterion & .32 & .31 & .31 & .23 & .12 & 1.59 & NS & NS \\
\hline Verbal STM & 80 & 83 & 91 & 90 & 96 & 13.03 & $\mathrm{p}<.001$ & $\begin{array}{l}8,9<10,11 \text {, adults } \\
\text { Adults }>\text { all child groups }\end{array}$ \\
\hline Spatial Span & 5.4 & 5.8 & 6.0 & 6.2 & 7.1 & 7.68 & $\mathrm{p}<.001$ & $\begin{array}{l}8<11 \& \text { adults } \\
\text { Adults }>\text { all child groups }\end{array}$ \\
\hline $\begin{array}{l}\text { Verbal executive } \\
\text { WM }\end{array}$ & 65 & 69 & 75 & 81 & 96 & 17.95 & $\mathrm{p}<.001$ & $\begin{array}{l}8<10,11 \text {, adults } \\
9<11, \text { adults } \\
\text { Adults }>\text { all child groups }\end{array}$ \\
\hline $\begin{array}{l}\text { SWM: Between } \\
\text { Search Errors } \\
\text { (across } 4,6,8 \text { box } \\
\text { difficulty levels) }\end{array}$ & 15.0 & 12.2 & 10.6 & 9.6 & 3.4 & 21.5 & $\mathrm{p}<.001$ & $\begin{array}{l}8<\text { all groups } \\
\text { adults }>\text { all child groups }\end{array}$ \\
\hline SWM: Strategy & 36 & 35 & 32 & 34 & 27 & 14.84 & $\mathrm{p}<.001$ & $\begin{array}{l}8<10 \& \text { adults } \\
9<10 \\
\text { Adults }>\text { all child groups }\end{array}$ \\
\hline $\begin{array}{l}\text { DMtS } \\
\quad \text { Simultaneous }(\%)\end{array}$ & 97 & 92 & 94 & 96 & 99 & NS & NS & NS \\
\hline $\begin{array}{l}\text { DMtS } \\
\text { Delay ( } \% \text { across } \\
\text { delays) }\end{array}$ & 72 & 77 & 80 & 87 & 92 & 10.6 & $\mathrm{p}<.001$ & $\begin{array}{l}8<10,11 \text {, adults } \\
9<\text { adults } \\
10<11 \text {, adults } \\
11>\text { all child groups } \\
\text { Adults }>8,9,10\end{array}$ \\
\hline
\end{tabular}

*Remember, Know, Guess, and Left/Right responses are corrected according to hits and false alarms (see statistical analysis section). 


\section{Correlational Data between Episodic Memory and Working Memory}

Bivariate Pearson correlations were conducted between key episodic memory and working memory measures on which developmental differences were reported. This involved the following episodic memory measures: hits for old responses, proportion of corrected Remember responses, proportion of corrected Know responses, and the following working memory measures: percentage of correct responses on the verbal STM component task, span length on the Spatial Span task, percentage of correct responses on the executive verbal WM task, total Between Search Errors and Strategy score on the SWM task, and percentage of correct responses across the delay conditions of the DMtS task. Correlations were conducted separately on each age of the five groups.

Table 3

Significant ( $p<0.01)$ correlations between key episodic and working memory measures

\begin{tabular}{|c|c|c|c|c|c|c|}
\hline Age & Measure & $\begin{array}{l}\text { Verbal } \\
\text { STM }\end{array}$ & $\begin{array}{l}\text { Spatial } \\
\text { Span }\end{array}$ & $\begin{array}{l}\text { Verbal } \\
\text { WM }\end{array}$ & $\begin{array}{l}\text { Spatial } \\
\text { WM }\end{array}$ & DMtS \\
\hline 8 & All N.S. & & & & & \\
\hline 9 & All N.S. & & & & & \\
\hline 10 & Hits & & & 0.59 & & 0.56 \\
\hline 10 & d-Prime & & & & & 0.63 \\
\hline 10 & Remember & & & & & \\
\hline 10 & Know & & & & & \\
\hline 11 & Hits & & & 0.52 & & \\
\hline 11 & d-Prime & & & 0.52 & & \\
\hline 11 & Remember & & & & & \\
\hline 11 & Know & & & & & -0.56 \\
\hline Adults & Hits & & & & -0.56 & \\
\hline Adults & d-Prime & & & & -0.56 & \\
\hline Adults & Remember & & & & & \\
\hline Adults & Know & & & & & \\
\hline
\end{tabular}

Correlational analyses on data for 8 and 9-year-olds revealed no significant relationships between episodic memory and WM measures. Analyses on data for the 10 and 11-year-olds revealed significant correlations between episodic memory performance and both the executive verbal WM task and delayed STM task. Analyses on data for 10 year olds revealed several significant correlations between accuracy on the episodic memory task and WM measures. Hit rate accuracy was positively related to accuracy on the executive verbal WM task ( $<<.01)$. 10-year-olds also showed significant positive correlations between hits and d-Prime and performance of the delay conditions of the DMtS task $(\mathrm{p}<.01)$. Analyses on data for 11-year-olds revealed significant correlations between accuracy and specific episodic retrieval measures and working memory measures. Hit rate accuracy and d-Prime were both positively related to accuracy on the executive verbal WM tasks $(\mathrm{p}<.01)$. Accuracy on the delay conditions of the DMtS task was negatively correlated with the proportion of Know responses. See Table 3.

Correlational analyses on the adult group data also revealed significant correlations between accuracy and specific episodic retrieval measures and WM measures, although in contrast to children these correlations were significant for the spatial executive WM task. Hit rate accuracy 
and d-prime were negatively correlated with errors on the SWM task in the adult group (p<.01), showing that for adults episodic memory performance relates to executive spatial WM.

\section{Discussion}

This study has revealed a distinct pattern of developmental changes in the engagement of episodic retrieval processes during the period of middle childhood. Unlike younger children, 11-year-olds engaged recollection to the same degree as adults. Developmental improvements in hit rate accuracy were observed on the episodic memory task; 8-year-old children were less accurate than 11-year olds and adults and indeed all child age groups with the exception of 11-year-olds were less accurate than adults. Inspection of the engagement of the retrieval processes of familiarity and recollection revealed that unlike children in all of the younger groups, 11-year-olds made a similar proportion of Remember responses to adults. Ten year olds in contrast showed an increased reliance on familiarity with more 'Know' responses than both 11-year-olds and adults. The present findings suggest that by age 11 children engage episodic retrieval processes in the same manner as adults. The study also reports developmental changes in verbal and spatial storage and executive aspects of working memory across middle childhood with all child age groups showing poorer performance to adults. In contrast, 11-year-olds performed similarly to adults on a delayed shortterm memory task requiring the ability to hold information in memory over a delay. Interestingly, children aged 8 and 9-years-old showed no significant relationships between episodic memory and working memory performance while 10 and 11-year-olds showed significant relationships between episodic memory measures and verbal working memory specific to the central executive component of verbal working memory. Adults showed a contrasting domain related pattern of correlations with significant relationships observed between episodic memory measures and spatial executive working memory.

The current findings add to the previous literature (Billingsley et al., 2002; Brainerd et al., 2004; Cycowicz et al., 2003; Defeyter et al., 2009; Ghetti and Angelini, 2008; Holliday et al., 2003) in identifying the specific ages at which changes in the engagement of episodic retrieval processes occur. Like Billingsley et al. the current study reports such changes using an episodic memory task that provides subjective measures of familiarity and recollection. Billingsley and colleagues (2002) reported that 8-10 year olds had a lower proportion of Remember responses than adolescent participant groups aged 14-16 and 17-19 years. Analysis of Know responses revealed that 8-10 year olds had a higher proportion of correct Know responses than the adolescent groups. The present study builds on these findings in showing significant changes in recollection and familiarity between the ages of 10 and 11 with a reduced reliance on familiarity and increased engagement of recollection. Importantly, the inclusion of a Guess response revealed that the youngest children (8-year-olds) who had shown the poorest hit rate on this task also made more Guess responses than older children and adults while 10 and 11-year-olds did not differ in proportion of Guess responses to adults, showing that younger children used the Guess option more frequently than older children and did not rely on Know responses for guessing. Surprisingly, 10-year-olds were the only age group to differ from 11-year-olds and adults in the proportion of Know responses they made. The inclusion of a Guess option may have encouraged 8 and 9-year-olds to choose Guess unless they were highly confident they had encountered the item before. In contrast, 10-year-old children, while showing the same levels of accuracy as 8 and 9year-olds, made few Guess responses and did not differ from older children and adults in this respect. It seems plausible that 10-year-olds may have been more confident than younger children that they encountered the item before and consequently made a higher proportion of Know 
responses. Further research using different methodological designs is warranted to clarify developmental trends in familiarity responding. That said, the current findings do suggest that a lack of significant differences reported in the Billingsley study in the proportion of Remember and Know responses between 8-10 year olds and 11-13 year olds may reflect power issues and Know responses at floor levels, rather than a lack of developmental changes during these time points. Previous ERP findings (Cycowicz et al., 2003) have suggested that children aged 9-10 and 12-13 rely on different neuronal networks when retrieving episodic information. The current findings show that future research incorporating imaging methodologies, tasks that incorporate both objective and subjective episodic retrieval measures, and comparison of child participants at specific ages during middle childhood and early adolescence is warranted.

The present study also assessed performance on a range of working memory measures namely working memory component and delayed short-term memory tasks, and related performance on the episodic memory task to functioning on these tasks. All child groups were less accurate than adults on verbal and spatial storage and central executive tasks. Correlational analyses revealed that younger children (aged 8 and 9 years) showed no significant relationships between episodic memory and working memory measures. In contrast, a specific relationship between episodic memory performance and executive aspects of verbal and spatial working memory was observed in older children (10,11 years) and adults. Interestingly, these relationships showed a domain difference between children and adults, with 10 and 11 year olds showing positive relationships between episodic memory and verbal executive working memory while adults showed this relationship with executive spatial working memory. While few studies have examined the relationship between working and episodic memory in children, a recently published study similarly reported a significant relationship between working memory capacity and quantity of information retrieved from long-term memory in children aged 9 and 11 (Roderer \& Roebers, 2009). The current study builds on this in suggesting a specific relationship between episodic memory and the executive component of working memory in children. The current study also reported that 8 and 9 -year-olds were less likely to use a strategy to aid performance on an executive working memory task (SWM task) than older children and adults. Collectively these findings suggest that use of strategies to aid memory improves during middle childhood and importantly these improvements are related to the ability to retrieve information from long-term memory. The study also reports that improvements in episodic memory during middle childhood are related to improvements in verbal executive working memory suggesting the possibility that use of verbal strategies is related to improved episodic memory performance.

A potential explanation for the lack of significant correlations between episodic memory and verbal executive working memory in 8 and 9-year-olds (unlike older children), is that children from 10 years onwards may sub-vocally label, and possibly rehearse, the study images hence relying on verbal executive working memory to support performance. It has previously been reported that at around the age of 8 years children begin to exhibit consistent word length effects and phonological similarity effects even when material is presented in a nonverbal form (Halliday \& Hitch, 1988). Interestingly, Marshall et al. (2002) interpreted laterality differences between child and adult ERP responses as reflecting the additional verbal naming strategies that they speculated adults engaged in when remembering pictorial items whereas they inferred children relied solely on non-verbal codes. The present study suggests the possibility that it is only round the age of 10 years that children use verbal information to aid memory performance on an episodic retrieval task. It would follow that a lack of such labeling in 8 and 9-year-olds may contribute to poorer retrieval of the image at test. Future research is warranted to investigate these possible relationships between verbal rehearsal and memory performance. It would be interesting for 
example to examine the performance of children in this age range on an episodic memory task with abstract images (which are not possible to label). There is indeed some evidence that private speech is related to performance on a cognitive planning task in 5 and 6-year-old children (Ferneyhough \& Fradley, 2005). Further research is required to address the specific role of private speech in aiding long-term memory in children.

The pattern of significant correlations observed between working memory and episodic memory is particularly interesting given the recent revision of Baddeley's working memory model to incorporate an episodic buffer (Baddeley, 2000; 2007). The current study reports that children and adult's episodic memory is selectively related to executive verbal and spatial working memory components with no significant relationships found between episodic memory and verbal and spatial short-term memory measures. This finding supports the original conception of the model which proposes that information is processed between the central executive and episodic long-term memory rather than via the verbal and spatial storage systems (Baddeley, 2000). The findings further suggest that children and adults may rely on differing executive strategies to support episodic retrieval. In contrast to children, adults in the current study showed significant correlations between episodic memory measures and executive spatial working memory suggesting that adults may use alternative strategies to children when engaging episodic memory. One possible explanation of the current study findings is that adults focus on using a strategy of remembering the location of the object on the screen at study over using a strategy to remember the specific item (i.e. as children may do with a verbal strategy) and hence in adults accuracy on the task relates significantly to visuo-spatial executive working memory. It should be noted that the episodic memory task employed in the current study was visual/spatial in nature. Future research can identify whether the same pattern of correlations between verbal and spatial working memory in children and adults would be observed using a verbal episodic task (e.g. involving auditory word lists). The verbal and spatial working memory tasks also differed considerably in relation to their response measures. The verbal tasks required a match/non-match decision while the spatial tasks involve a reproduction of the to-be-remembered item (Spatial Span) and a complex search task (Spatial Working Memory). These task differences may have influenced the correlations reported. Nonetheless, it is interesting that children and adults showed a differential set of relationships between the current visual/spatial episodic task and aspects of working memory and all were assessed on the same tasks. It therefore seems plausible that children and adults may rely on different strategies to aid memory retrieval.

Developmental improvements were also observed in the ability to hold information in memory over a delay (DMtS task), but for this measure 11-year-olds performed at adult levels. Ten and eleven year olds showed significant correlations between episodic memory accuracy and Know responses and accuracy on this delayed short-term memory task. The findings presented here suggest that the ability to hold information over a delay may relate to developmental changes in reduced reliance on familiarity when retrieving information from episodic memory.

Interestingly, unlike their performance on all other working memory measures, 11-year-olds did not differ from adults in performance on the delayed short-term memory task supporting the idea that the ability to hold information in memory over time is important to developmental improvements in long-term memory retrieval.

While the design of the current study has enabled the comparison of episodic memory and working memory performance at specific time points during middle childhood the study is limited by the narrow age range. Other studies which have incorporated wider age spans suggest developmental changes in the engagement of recollection and familiarity from earlier in middle childhood into adolescence (e.g. Ghetti \& Angelini, 2008). While we have been able to show that 
11-year-olds perform at adult levels on the episodic memory task, they did show significantly poorer performance in verbal and spatial storage and executive working memory to adults. Investigation of the relationship between episodic retrieval and working memory into adolescence is thus warranted to fully investigate the developmental changes in the relationship between these short and long-term memory systems. Furthermore, it is well established that children show developmental changes across the period of middle childhood and early adolescence in a wide variety of aspects of executive function beyond working memory to include inhibition, attentional flexibility, and planning (e.g. Luciana \& Nelson, 1998). A number of source memory studies have indeed reported relationships between source memory accuracy and aspects of executive functioning including verbal fluency and attentional flexibility (e.g. Drummey \& Newcombe, 2002; Rybash and Colilla, 1994; Sluzenski, Newcombe, \& Ottinger 2004). The current findings indeed suggest that children's use of strategies is related to retrieving information about episodes they have experienced in the past. Future research endeavours can facilitate broader insights into the relationship between the development of executive functions beyond working memory and the specific engagement of episodic retrieval processes.

In conclusion, the findings support previous findings of improvements in episodic retrieval processes during middle childhood and suggest that by age 11 children engage episodic retrieval processes in the same manner as adults. The findings also suggest that age-related improvements in episodic memory may relate to specific underlying changes in verbal executive aspects of working memory and improvements in the ability to hold information in short-term memory over a delay.

\section{References}

Atkinson, R.C. \& Joula, J.F. (1973). Factors influencing speed and accuracy of word recognition. In S. Kornblum (Ed.), Fourth international symposium on attention and performance (pp. 583-611). New York: Academic Press.

Baddeley, A.D. (1986). Working memory. Oxford: Clarendon Press.

Baddeley, A.D. (2000). The episodic buffer: A new component of working memory? Trends in Cognitive Sciences, 4, 417-423.

Baddeley, A.D. (2007). Working memory, thought and action. Oxford University Press.

Baddeley, A.D. \& Hitch, G. (1974). Working Memory, In G. Bower (Ed.), The psychology of learning and motivation, 8, pp 47-90. New York: Academic Press.

Billingsley, R.L., Smith, M.L., \& McAndrews, M.P. (2002). Developmental patterns in priming and familiarity in explicit recollection. Journal of Experimental Child Psychology, 82, 251277.

Brainerd, C.J., Holliday, R.E., \& Reyna, V.F. (2004). Behavioural measurement of remembering phenomenologies: so simple a child can do it. Child Development, 75, 2, 505-522.

Cannon, T.D., Glahn, D.C., Kim, J., Van Erp, T.G.M., Karlsgodt, K., Cohen, M.S., Nuechterlein, K.H., Bava, S., \& Shirinyan, D. (2005). Dorsolateral prefrontal cortex activity during maintenance and manipulaton of information in working memory in patients with schizophrenia. Archives of General Psychiatry, 62, 1071-1080.

Curtis, W.J., Lindeke, L.L., Georgieff, M.K., Nelson, C.A. (2002). Neurobehavioural functioning in neonatal intensive care unit graduates in late childhood and early adolescence. Brain $125,7,1646-1659$.

Cycowicz, Y.M., Friedman, D., Snodgrass, J.G. \& Duff, M. (2001). Recognition and source memory for pictures in children and adults. Neuropsychologia, 39, 255-267. 
Cycowicz, Y.M., Friedman, D. \& Duff, M. (2003). Pictures and their colours: what do children remember. Journal of Cognitive Neuroscience, 15, 5, 759-768.

Defeyter, M.A., Russo, R. \& McPartlin, P.L. (2009). The picture superiority effect in recognition memory: A developmental study using the response signal procedure. Cognitive Development, 24, 265-273.

D’Esposito, M., Postle, B.R., Ballard, D., Lease, J., (1999). Maintenance versus manipulation of information held in working memory: an event-related fMRI study. Brain and Cognition 41, 66- 86.

Drummey, A.B. \& Newcombe, N.S. (2002). Developmental changes in source memory. Developmental Science, 5, 4, 502-513.

Dunn, L., Dunn, L., Whetton, C., \& Burley, J. (1997). British Picture Vocabulary Scale II. GL Assessment: London.

Ferneyhough, C. \& Fradley, E. (2005). Private speech on an executive task: relations with task difficulty and task performance. Cognitive Development, 20, 103-120.

Foley, M.A., Johnson, M.K., \& Raye, C.L. (1983). Age-related changes in confusion between memories for speech and memories for thought. Child Development, 54, 510-560.

Foley, M.A. \& Johnson, M.K., (1985). Confusions between memories for performed and imagined actions: a developmental comparison. Child Development, 56, 1145-1155.

Fray, P.J., \& Robbins, T.W. (1996). CANTAB battery: proposed utility in neurotoxicology. Neurotoxicology and Teratology, 18, 499-504.

Gardiner, J. M. \& Richardson-Klavehn, A. (2000). Remembering and knowing. In E. Tulving \& F. I. M. Craik (Eds.), The Oxford handbook of memory (pp. 229-244). New York: Oxford University Press.

Ghetti, S. \& Angelini, L. (2008). The development of recollection and familiarity in childhood and adolescence: Evidence from the dual process signal detection model. Child Development, 79, 2, 339-358.

Goodman, R. (2001). Psychometric properties of the strengths and difficulties questionnaire, Journal of the American Academy of Child and Adolescent Psychiatry, 40, 11, 1337-1345.

Halliday, M.S. \& Hitch, G.J. (1988). Developmental applications of working memory. In: G. Claxton, Editor, New directions in cognition, Routledge \& Kegan Paul, London, pp. 193222.

Holliday, R.E. (2003). Reducing misinformation effects in children with cognitive interviews: dissociating recollection and familiarity. Child Development, 74, 3, 728-751.

Jacoby, L.L. (1991). A process dissociation framework: Separating automatic from intentional uses of memory. Journal of Memory and Language, 30, 5, 513-541.

Jacoby, L.L., Jennings, J.M., \& Hay, J.F. (1996). Dissociating automatic and consciously controlled processes: implications for diagnosis and rehabilitation of memory deficits. In D.J.

Kim, J., Glahn, D.C., Nuechterlein, K.H., Cannon, T.D. (2004). Maintenance an manipulation of information in schizophrenia: further evidence for impairment in the central executive component of working memory. Schizophrenia Research, 68, 173-187.

Knott, L.M., \& Dewhurst, S.A. (2007). Divided attention at retrieval disrupts knowing but not remembering. Memory, 15, 664-674.

Koriat, A. \& Goldsmith, M. (1996). Monitoring and control processes in the strategic regulation of memory accuracy. Psychological Review, 103, 3, 490-517.

Luciana,M. \& Nelson,C.A. (1998). The functional emergence of prefrontally-guided working memory systems in four- to eight-year-old children. Neuropsychologia 36, 3, 273-293. 
Mandler, G. (1980). Recognizing: The judgment of previous occurrence. Psychological Review, $87(3), 252-271$.

Marshall, D.H., Drummey, A.B., Fox, N.A., \& Newcombe, N.S. (2002). An event-related potential study of item recognition memory in children and adults. Journal of Cognition and Development, 3, 2, 201-224.

Milner, B., Corsi, P., \& Leonard, G. (1991). Frontal-lobe contribution to recency judgements. Neuropsychologia, 29, 601-618.

Miyake, A., Friedman, N. P., Rettinger, D. A., Shah, P., \& Hegarty, M. (2001). How are visuospatial working memory, executive functioning, and spatial abilities related? A latent variable analysis. Journal of Experimental Psychology: General, 130, 621-640.

Morris, R.G., Evendon, J.L., Sahakian, B.J. \& Robbins, T.W. (1987). Computer-aided assessment of dementia: comparative studies of neuropsychological deficits in Alzheimers-type dementia and Parkinson's disease. In S.M. Stahl, S.D. Iverson \& E.C. Goodman (Eds.) Cognitive Neurochemistry. Oxford University Press: Oxford pp21-36.

Petrides, M., \& Milner, B. (1982). Deficits on subject-ordered tasks after frontal- and temporal-lobe lesions in man. Neuropsychologia, 20, 249-262.

Ranganath, C., Cohen, M.X. \& Brozinsky, C.J. (2005). Working memory maintenance contributes to long-term memory formation: neural and behavioural evidence. Journal of Cognitive Neuroscience, 17, 7, 994-1010.

Rhodes, S. M., Coghill, D. R., \& Matthews, K. (2004). Methylphenidate restores visual memory, but not working memory function in attention deficit-hyperkinetic disorder. Psychopharmacology, 175, 319-330.

Rhodes, S. M., Coghill, D. R., \& Matthews, K. (2005). Neuropsychological functioning in stimulant-naive boys with hyperkinetic disorder. Psychological Medicine, 35, 1109-1120.

Rhodes, S. M., Coghill, D. R., \& Matthews, K. (2006). Acute neuropsychological effects of methylphenidate in stimulant drug-naïve boys with ADHD II - broader executive and non-executive domains. Journal of Child Psychology and Psychiatry 47, 11, 1184-1194.

Rhodes, S.M. \& Donaldson, D.I. (2007). Electrophysiological evidence for the influence of unitization on the processes engaged during episodic retrieval: enhancing familiarity based remembering. Neuropsychologia, 45, 412-424.

Rhodes, S.M. and Donaldson, D.I. (2008). Electrophysiological evidence for the effect of interactive imagery on episodic memory: encouraging familiarity for non-unitized stimuli during associative recognition. Neuroimage, 39, 2, 873-884.

Robbins, T.W., James, M., Owen, A.M., Sahakian, B.J., McInnes, L., \& Rabbitt, P. (1994). Cambridge Neuropsychological Test Automated Battery (CANTAB): a factor analytic study of a large sample of normal elderly volunteers. Dementia, 5, 266-281.

Roderer, T. \& Roebers, C.M. (2009). Children's strategic regulation of memory accuracy. In Applied Memory, pp253-274. Edited by M.R. Kelley.

Rybash, J.M. \& Colilla, J.L. (1994). Source memory deficits and frontal lobe functioning in children. Developmental Neuropsychology, 10, 67-73.

Shah, P., \& Miyake, A. (1996). The separability of working memory resources for spatial thinking and language processing: An individual differences approach. Journal of Experimental Psychology. General, 125, 4-27.

Sluzenski, J., Newcombe, N.S., \& Ottinger, W. (2004). Changes in reality monitoring and episodic memory in early childhood. Developmental Science, 7, 2, 225-245.

Sluzenski, J., Newcombe, N.S. \& Kovacs, S.L. (2006). Binding, relational memory, and recall 
of naturalistic events: a developmental perspective. Journal of Experimental Psychology: Learning, Memory and Cognition, 32, 1, 89-100.

Snodgrass, J.G. \& Vanderwart, M. (1980). A standardized set of 260 pictures: norms for name agreement, image agreement, familiarity, and visual complexity. Journal of Experimental Psychology, Human, Learning, and Memory, 6, 174-215.

Tulving, E. (1985). Memory and consciousness. Canadian Psychologist, 26, 1-12.

Welsh, M.C., Pennington, B.F., \& Grossier, D.B. (1991). A normative-developmental study of executive function: a window of prefrontal function in children. Developmental Neuropsychology, 7, 131-149.

Wilding E.L. \& Rugg, M.D. (1996). An Event-Related Potential Study of Recognition Memory With and Without Retrieval of Source. Brain, 119, 889-905.

Yonelinas, A.P. (2002). The nature of recollection and familiarity: A review of 30 years of research. Journal of Memory and Language, 46, 441-517. 